\title{
Prognostic significance of postoperative longitudinal change of serum carcinoembryonic antigen level in patients with stage I lung adenocarcinoma completely resected by single-port video-assisted thoracic surgery: a retrospective study
}

\author{
Hao Chen ${ }^{1 \#} \wedge$, Yan Jiang ${ }^{1 \#}$, Keyi Jia ${ }^{2 \#}$, Kaixuan Zhang ${ }^{1}$, Natsumi Matsuura ${ }^{3}$, Jin Yong Jeong ${ }^{4}$, Bo Su ${ }^{5}$, \\ Xiao Zhou ${ }^{1}$
}

${ }^{1}$ Department of Thoracic Surgery, Shanghai Pulmonary Hospital, Tongji University School of Medicine, Shanghai, China; ${ }^{2}$ Department of Medical Oncology, Shanghai Pulmonary Hospital, Tongji University School of Medicine, Shanghai, China; ${ }^{3}$ Department of General Thoracic Surgery, Japanese Red Cross Maebashi Hospital, Gunma, Japan; ${ }^{4}$ Department of Thoracic and Cardiovascular Surgery, Incheon St. Mary's Hospital, College of Medicine, The Catholic University of Korea, Incheon, Republic of Korea; ${ }^{5}$ Central Laboratory, Shanghai Pulmonary Hospital, Tongji University School of Medicine, Shanghai, China

Contributions: (I) Conception and design: H Chen, X Zhou; (II) Administrative support: X Zhou, B Su; (III) Provision of study materials or patients: X Zhou; (IV) Collection and assembly of data: H Chen, Y Jiang; (V) Data analysis and interpretation: H Chen, K Jia; (VI) Manuscript writing: All authors; (VII) Final approval of manuscript: All authors.

\#These authors contributed equally to this work.

Correspondence to: Xiao Zhou. Department of Thoracic Surgery, Shanghai Pulmonary Hospital, Tongji University School of Medicine, No. 507 Zhengmin Road, Yangpu District, Shanghai 200433, China. Email: zx_shfk68@163.com; Bo Su. Central Laboratory, Shanghai Pulmonary Hospital, Tongji University School of Medicine, No. 507 Zhengmin Road, Yangpu District, Shanghai 200433, China. Email: su_bo_s@hotmail.com.

Background: Patients with stage I lung adenocarcinoma (LUAD) have varying postoperative prognosis. This study aimed to investigate the prognostic significance of postoperative longitudinal change of serum carcinoembryonic antigen (CEA) level in patients with stage I LUAD.

Methods: The study cohort comprised 241 patients with stage I LUAD completely resected with singleport video-assisted thoracic surgery (VATS). The patients were categorized into 4 groups according to the postoperative longitudinal change of serum CEA levels measured in the third and sixth months after surgery: the NN group (continuously normal), HN group (increase first and then decrease), NH group (decrease first and then increase), and HH group (continuously high). Recurrence-free survival (RFS) was analyzed by the Kaplan-Meier method and compared by log-rank test. A nomogram was developed to predict recurrence in the stage I LUAD patients.

Results: In univariate analysis, differentiation $(\mathrm{P}<0.001)$, visceral pleural invasion (VPI) $(\mathrm{P}=0.025)$, tumor diameter $(\mathrm{P}<0.001)$, tumor-node-metastasis $(\mathrm{TNM})$ stage $(\mathrm{P}=0.008)$, preoperative CEA levels $(\geq 10.0$ vs. $<10.0 \mathrm{ng} / \mathrm{mL}, \mathrm{P}<0.001)$, and postoperative CEA grouping $(\mathrm{NH} / \mathrm{HH}$ vs. $\mathrm{NN} / \mathrm{HN}, \mathrm{P}<0.001)$ were significant prognostic factors for stage I LUAD patients. Multivariate analysis showed that tumor diameter $(\mathrm{P}=0.009)$ and postoperative CEA grouping $(\mathrm{P}<0.001)$ were considered to be independent prognostic factors of postoperative recurrence of stage I LUAD. Tumor diameter $(\geq 20 \mathrm{~mm})$ and postoperative CEA (NH/HH vs. NN/HN) were associated with worse RFS. Receiver operating characteristic (ROC) curve analysis showed that postoperative CEA (NH/HH vs. NN/HN) have high sensitivity (64.7\%) and specificity (83.2\%) for early prediction of postoperative recurrence of stage I LUAD. The area under curve (AUC) value was 0.745. The nomogram based on multivariate Cox regression had a concordance index (value of 0.789). The calibration plot showed that the predicted probabilities closely matched the observed probabilities.

$\wedge$ ORCID: 0000-0002-6127-8773. 


\begin{abstract}
Conclusions: Longitudinal change in serum CEA level after surgery was found to be an independent unfavorable prognostic factor in completely resected stage I LUAD patients. The NH group and HH group were significantly associated with worse RFS. A nomogram was established to predict the postoperative recurrence of patients with stage I LUAD.
\end{abstract}

Keywords: Lung adenocarcinoma (LUAD); postoperative; carcinoembryonic antigen (CEA); minimal residual disease (MRD); longitudinal

Submitted Aug 26, 2021. Accepted for publication Oct 20, 2021.

doi: $10.21037 /$ tlcr-21-833

View this article at: https://dx.doi.org/10.21037/tlcr-21-833

\section{Introduction}

Lung cancer remains the leading cause of cancer-related death worldwide, and non-small cell lung cancer (NSCLC) accounts for the greatest proportion of these lung cancer cases $(1,2)$. The standard treatment for resectable NSCLC is anatomic resection plus mediastinal lymph node dissection $(3,4)$. Lung adenocarcinoma (LUAD) is currently the one of NSCLC subtypes with the highest incidence and involves a higher possibility of developing micrometastasis in patients with early-stage cancer (5). In fact, even if the local treatment of complete resection is successful, the recurrence rate is still high after surgery (6-9), and postoperative recurrence is the main cause of death in stage I NSCLC (10-12). Some studies have suggested that recurrence is caused by minimal residual disease (MRD) $(13,14)$. A large-scale study in Japan reported a 5 -year recurrence-free survival (RFS) rate for stage I lung cancer of $65.8-84.3 \%$ (15), suggesting the presence of other prognostic factors besides disease stage and micrometastases. Therefore, close monitoring is needed to detect recurrence in stage I LUAD patients after surgery as early as possible and thus improve patient outcomes.

Low-dose computed tomography (LDCT) is crucial for the early screening and postoperative monitoring of lung cancer (16-18). Unfortunately, the medical follow-up of imaging surveillance after surgery is low, and it is not easy to detect early recurrence $(19,20)$. Additionally, LDCT screening has some limitations, including a high rate of false positives, radiation-related risk, and high cost $(21,22)$. Therefore, a simple, convenient, and accurate method for monitoring the postoperative recurrence of lung cancer is urgently needed.

CEA is a member of the immunoglobulin supergene family (IGSF) and may play a role in tumorigenesis by enhancing the binding between tumor cells and normal cells in LUAD (23). Carcinoembryonic antigen (CEA) is an important tumor marker and has been found to be elevated in a variety of malignant tumors (24-27). The expression of CEA also increases in patients with lung cancer, especially those with LUAD, and has higher diagnostic value than do the other types (28-30). Most relevant current studies have focused on either the predictive effect of the preoperative serum CEA level on the prognosis of patients or on combining serum CEA with other markers for diagnosis (31-36). These reports are only the results of a one-time preoperative CEA test. However, our study used the combination of the preoperative CEA level and the longitudinal changes of the two postoperative CEA level, which could allow us to get more information. Studies on the longitudinal change of serum CEA after surgery are still rare. The CEA was also found to be elevated in a variety of malignant tumors after surgery. the elevated postoperative CEA were at increased risk for recurrence in colon cancer (37) and associated with shorter OS in gastric cancer (38). The study of longitudinal change is a systematic and regular study of the same object over a relatively long period of time. It could see a more complete development process and some key turning points in the development process. Consequently, we conducted a study to examine the postoperative longitudinal change of serum CEA level in patients with stage I LUAD to establish a model that can predict recurrence in the early postoperative period. We present the following article in accordance with the TRIPOD reporting checklist (available at https://dx.doi. org/10.21037/tlcr-21-833).

\section{Methods}

\section{Patients and samples}

The hospital records of 1,341 patients who underwent single-port video-assisted thoracic surgery (VATS) resection 
of stage I LUAD at Shanghai Pulmonary Hospital, Tongji University from August 2015 to January 2016 were retrospectively analyzed and followed up until January 2021. The serum level of CEA was measured as a part of the routine preoperative evaluation within 2 days prior to surgery. The selection criteria for patients were as follows: (I) complete CEA test at 3 and 6 months after surgery (in addition to the need for rest after the operation, it can also avoid CEA fluctuations caused by surgical injuries within one month), (II) no autoimmune diseases or other primary tumors, and (III) pathologically confirmed stage I LUAD according to the eight edition of the Union for International Cancer Control (UICC) staging. The exclusion criteria were the following: (I) patients who received chemotherapy, biological therapy, or immunotherapy before surgery; (II) patients with severe heart disease, tuberculosis, or infections; and (III) patients who were lost to follow-up. This study was carried out in accordance with the principles of the World Medical Association's Declaration of Helsinki (as revised in 2013). The study was approved by Shanghai Pulmonary Hospital Institutional Review Board (No. KY2020-1). Patient's informed consent was waived due to the retrospective nature of this study. After meeting the inclusion and exclusion criteria, 241 patients were enrolled into the study (Figure S1). Disease stages were based on the eighth edition of the American Joint Committee on Cancer staging manual. Operations were performed as single-port VATS. The characteristics of the patients were collected and are summarized in Table 1 .

Serum CEA level was measured 2 days before the operation and at 3 and 6 months after the operation by an automated electrochemiluminescence analyzer (Beijing Tigsun Diagnostics Co., Ltd., Beijing, China) in accordance with the manufacturer's instructions. The cutoff value of CEA uses the reference value of the kit. The upper limit of normal in our hospital was defined as $10 \mathrm{ng} / \mathrm{mL}$ based on the $95 \%$ specificity level.

RFS was defined as the time from the date of surgery to recurrence or death or the date of the last follow-up. The follow-up period was from August 2015 to January 2021. When the level of serum CEA was higher than that measures previously or exceeded the upper limit of the normal value, it was defined as " $\mathrm{H}$ " (higher); otherwise, it was defined as " $\mathrm{N}$ " (normal, no higher). The patients were divided into 4 groups according to the longitudinal change of the 2 CEA levels measured in the third and sixth months after surgery: the NN group (continuously normal), $\mathrm{HN}$ group (increase first and then decrease), $\mathrm{NH}$ group (decrease first and then increase), and $\mathrm{HH}$ group (continuously high). Follow-up information was obtained from all patients through telephone interviews either with the patient or with a relative.

\section{Statistical analysis}

Categorical variables were compared by using Fisher's exact test, correlation test, or chi-square test. The patients' RFS was analyzed by the Kaplan-Meier method and compared by the log-rank method. A multivariate Cox regression model was used to test the independent factors related to RFS, and the hazard ratios (HRs) were calculated. A nomogram was conducted based on the multivariate Cox regression model using $\mathrm{R}$ package (the $\mathrm{R}$ Foundation for Statistical Computing). Receiver operating characteristic (ROC) analysis was performed to determine the cutoff value of independent predictors to predict disease recurrence in stage I LUAD patients. All P values were based on a twosided hypothesis. SPSS 22.0 software (IBM Corporation, Armonk, NY, USA) was used for all analyses.

\section{Results}

\section{Characteristics of stage I LUAD patients}

The demographic and clinicopathological characteristics of 241 patients with stage I LUAD are shown in Table 1. The study included 108 (44.8\%) males and 133 (55.2\%) females. The average age of patients was 59.5 years (range, 24-82 years) The median follow-up time was 62 months with a range of 7-65 months.

According to the longitudinal change of the postoperative level of serum CEA measured twice after the operation, the patients were divided into 4 groups: the NN group (continuously normal, $70.1 \%$ ), HN group (increase first and then decrease, $2.9 \%$ ), $\mathrm{NH}$ group (decrease first and then increase, $19.5 \%$ ), and HH group (continuously high, $7.5 \%)$. These 4 groups showed no statistical differences with respect to gender, age ( $<60 v s . \geq 60$ years), smoking history, tumor-node-metastasis (TNM) stage, lesion location, or preoperative CEA level. $14.9 \%$ of patients with stage I LUAD had a preoperative CEA level that exceeded the normal cutoff $(\geq 10 \mathrm{ng} / \mathrm{mL})$. Postoperative CEA group was significantly correlated with tumor diameter $(\mathrm{P}=0.010)$, differentiation $(\mathrm{P}<0.001)$, visceral pleural invasion (VPI) $(\mathrm{P}=0.031)$, and recurrence $(\mathrm{P}<0.001)$.

Recurrence occurred in 51 (21.2\%) of the 241 patients, with the $\mathrm{NN}$ group, $\mathrm{HN}$ group, $\mathrm{NH}$ group, and $\mathrm{HH}$ group 
Table 1 Clinical characteristics of the 241 patients with stage I LUAD

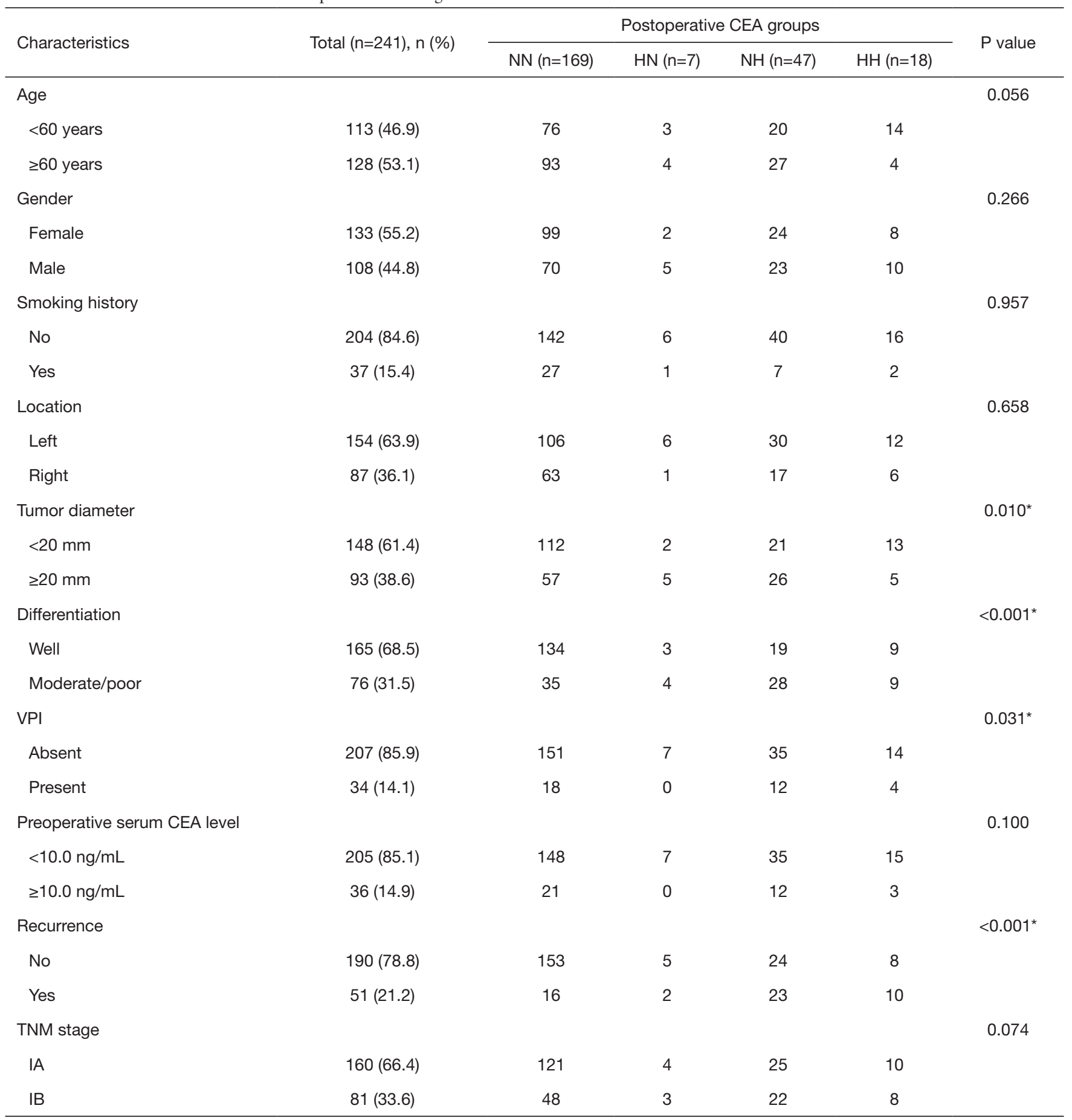

Longitudinal change of postoperative CEA: NN, continuously normal; $\mathrm{HN}$, increase first and then decrease; $\mathrm{NH}$, decrease first and then increase; $\mathrm{HH}$, continuously high. * $\mathrm{P}<0.05$. LUAD, lung adenocarcinoma; CEA, carcinoembryonic antigen; VPI, visceral pleural invasion; TNM, tumor-node-metastasis; LUAD, lung adenocarcinoma. 
A

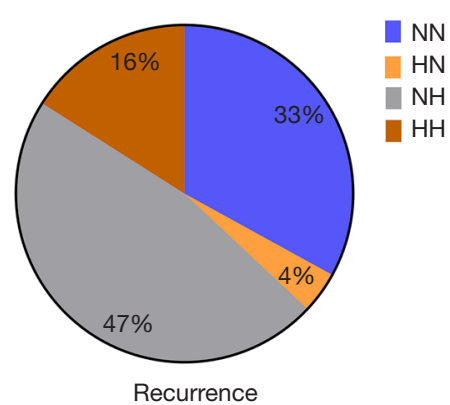

B

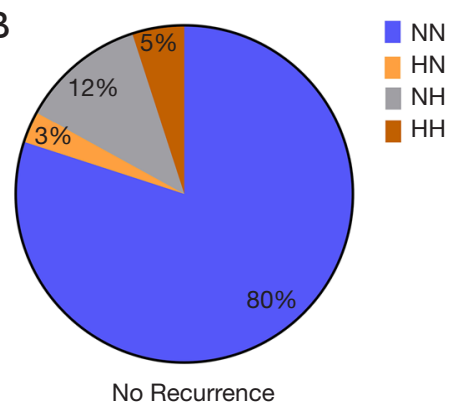

C

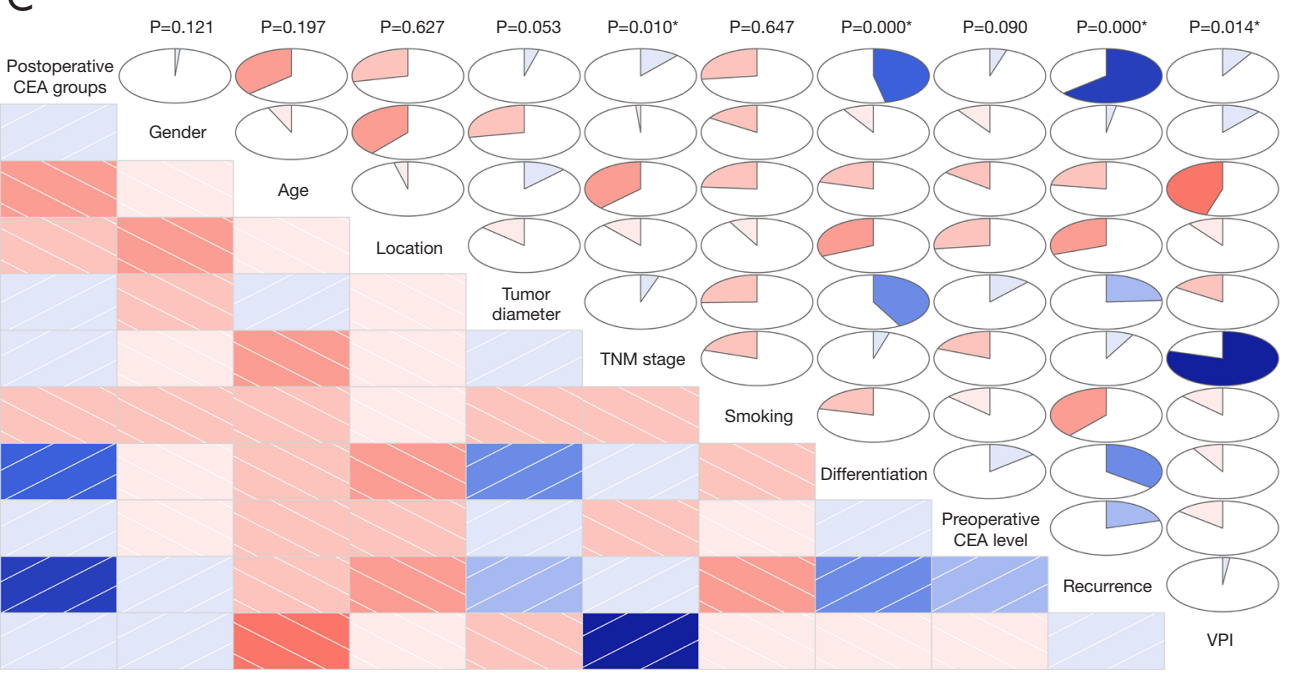

Figure 1 Correlations between postoperative CEA groups and clinicopathological characteristics of stage I LUAD patients. (A) Spearman correlation coefficient was used to analyze the correlation between the postoperative CEA groups and the clinicopathological characteristics of 241 stage I LUAD patients. (B) In recurrence, the proportion of different postoperative CEA groups. (C) In no recurrence, the proportion of different postoperative CEA groups. Longitudinal change of postoperative CEA: NN, continuously normal; HN, increase first and then decrease; $\mathrm{NH}$, decrease first and then increase; $\mathrm{HH}$, continuously high. * $\mathrm{P}<0.05$. CEA, carcinoembryonic antigen; VPI, visceral pleural invasion; TNM, tumor-node-metastasis; LUAD, lung adenocarcinoma.

accounting for $33 \%, 4 \%, 47 \%$, and $16 \%$ of the recurrence cases, respectively (Figure 1A). Conversely, the $\mathrm{NN}$ group, $\mathrm{HN}$ group, $\mathrm{NH}$ group, and $\mathrm{HH}$ group, accounted for $80 \%$, $3 \%, 12 \%$, and $5 \%$, of the non-recurrence cases, respectively (Figure 1B). The proportion of $\mathrm{NH}$ and $\mathrm{HH}$ patients with recurrence was significantly higher than that of nonrecurrence patients (63\% vs. $17 \%)$. We found that the longitudinal change of CEA level in patients who recurred after surgery was more likely to involve an increase.

\section{Correlation between postoperative serum CEA grouping and clinical characteristics}

In order to further explore the relationship between postoperative CEA grouping and the clinical characteristics of stage I LUAD, we used the Spearman correlation coefficient (Figure 1C). After analysis, we found that there was no significant statistical correlation between postoperative CEA grouping and gender $(\mathrm{P}=0.121)$, age $(\mathrm{P}=0.197)$, location $(\mathrm{P}=0.627)$, tumor diameter $(\mathrm{P}=0.053)$, or smoking history $(\mathrm{P}=0.647)$. Postoperative CEA grouping was only associated with pathological TNM (pTNM; $\mathrm{P}=0.010)$, differentiation $(\mathrm{P}<0.001)$, recurrence $(\mathrm{P}<0.001)$, and VPI $(\mathrm{P}=0.014)$.

\section{Univariate and multivariate Cox regression for predicting the RFS of stage I LUAD patients}

Univariate Cox analysis, performed on the 241 patients with 
stage I LUAD, demonstrated that degree of differentiation (well/moderate and poor; $\mathrm{P}<0.001$ ), VPI (absent/present; $\mathrm{P}=0.025)$, tumor diameter $(<20 / \geq 20 \mathrm{~mm} ; \mathrm{P}<0.001)$, TNM stage (IA/IB; $\mathrm{P}=0.008$ ), preoperative CEA levels $(<10.0 / \geq 10.0 \mathrm{ng} / \mathrm{mL} ; \mathrm{P}<0.001)$, and postoperative CEA grouping ( $\mathrm{NN} v s$. $\mathrm{NH}$; $\mathrm{NN}$ vs. $\mathrm{HH}$; both $\mathrm{P}<0.001)$ were significant prognostic factors (Table 2).

Using the above variables with significant prognostic significance and gender variables, we performed multivariate Cox regression analysis. The results indicated that the independent influencing factors of RFS were tumor diameter [hazard ratio (HR): 2.437 ; $95 \%$ confidence interval (CI): 1.245-4.770; $\mathrm{P}=0.009$ ] and postoperative CEA grouping (NN vs. NH; HR: 4.672; 95\% CI: 2.294-9.514, $\mathrm{P}<0.001$; NN vs. HH; HR: 10.266; 95\% CI: 4.452-23.673; $\mathrm{P}<0.001)$. Kaplan-Meier survival analysis with a log-rank test showed that postoperative CEA grouping (Figure 2A) and tumor diameter (Figure $2 B$ ) had the best predictive value for RFS in stage I LUAD patients. The postoperative 5 -year RFS rate of patients in the NN group, HN group, $\mathrm{NH}$ group, and $\mathrm{HH}$ group was $90.5 \%, 71.4 \%, 50.1 \%$, and 44.4, respectively. Therefore, tumor diameter $(\geq 20 \mathrm{~mm})$ and postoperative CEA $(\mathrm{NH}, \mathrm{HH})$ groups were considered to be important independent predictors of postoperative recurrence of stage I LUAD.

\section{Diagnostic value evaluation of preoperative serum CEA levels and postoperative serum CEA grouping for stage I LUAD}

According to the 5-year RFS rate of patients in the NN group, $\mathrm{HN}$ group, $\mathrm{NH}$ group and $\mathrm{HH}$ group, we divided the results from high to low into 4 grade variables from small to large: $\mathrm{I}=\mathrm{NN}, \mathrm{II}=\mathrm{HN}, \mathrm{III}=\mathrm{NH}$, and $\mathrm{IV}=\mathrm{HH}$. Additionally, we divided the patient's preoperative serum CEA levels into 2 categories: $<10.0 \mathrm{ng} / \mathrm{mL}$, normal group; and $\geq 10.0 \mathrm{ng} / \mathrm{mL}$, abnormal group. Receive operating characteristic (ROC) analysis was used to determine the values of preoperative serum CEA levels and the postoperative serum CEA groups in the diagnosis of RFS in stage I LUAD patients. The area under curve (AUC) value was 0.754 for the postoperative serum CEA group (Figure 2C) and 0.592 for the preoperative serum CEA level group (Figure 2D). Based on a variety of sensitivity and misdiagnosis rates on ROC, the Youden index (YI) was used to calculate the best cutoff value for the 2 above-mentioned variables for early diagnosis of postoperative recurrence of stage I LUAD. We defined the values of postoperative serum CEA groups $\geq$ III (NH group and HH group) as positive. The specificity and sensitivity of the 2 indicators for early diagnosis of postoperative recurrence of stage I LUAD were calculated and compared with pathological diagnosis. The sensitivity and specificity were $29.4 \%$ and $88.9 \%$ for preoperative serum CEA levels and $64.7 \%$ and $83.2 \%$ for postoperative serum CEA grouping, respectively. This demonstrated that postoperative serum CEA grouping has high sensitivity and specificity for early diagnosis of postoperative recurrence of stage I LUAD.

\section{Nomogram and calibration}

On the basis of the multivariate Cox regression, a nomogram (Figure $3 A$ ) was developed to calculate the 1-, 3-, and 5-year RFS probability. The calibration plot showed that the predicted probabilities closely matched the observed probabilities. The concordance index (C-index) value of the prediction model was 0.789 . Validation indicated that this nomogram exhibited excellent predictive ability and calibration (Figure 3B-3D).

\section{Discussion}

Lung cancer is among the most prevalent and lethal cancers worldwide, with the most common pathological type of lung cancer being LUAD (1). With the increasing popularity of imaging screening (LDCT, positron emission tomographyCT, etc.), especially in China, LUADs are being found with greater frequency in the early stages of the disease $(16,39)$. Surgery still remains the only curative therapy for completely resectable stage I LUAD $(3,4)$. However, a Japanese study found that even among patients in the same stage I LUAD, the prognosis was different (15). Presumably other influencing factors exist, such as MRD and TNM stage. LDCT has been the most important method for early screening and postoperative monitoring of lung cancer (16-18). However, LDCT screening has several limitations, including its inability to detect early recurrence, high false positive rate, radiationrelated risk, and high costs (19-22). Therefore, we must not only explore other possible important influencing factors, but also establish a simple, safe, and efficient monitoring method to detect potential recurrence risks in stage I LUAD as soon as possible after surgery.

First discovered by Gold and Freedman in 1965 (40), CEA is an acid glycoprotein with human embryonic antigen specificity present in the human digestive system and embryonic tissues. CEA has been studied in gastric cancer (24), 
Table 2 Predictors of RFS according to univariate and multivariate Cox regression analysis

\begin{tabular}{|c|c|c|c|c|c|c|}
\hline Characteristic & \multicolumn{3}{|c|}{ Univariable analysis } & \multicolumn{3}{|c|}{ Multivariable analysis } \\
\hline \multicolumn{7}{|l|}{ Age } \\
\hline$<60$ years & 1.000 & Reference & & & & \\
\hline$\geq 60$ years & 1.045 & $0.603-1.811$ & 0.875 & & & \\
\hline Male & 1.000 & Reference & & 1.000 & Reference & \\
\hline Female & 1.723 & $0.990-3.000$ & 0.054 & 1.301 & $0.720-2.351$ & 0.384 \\
\hline \multicolumn{7}{|l|}{ Smoking history } \\
\hline Yes & 1.000 & Reference & & & & \\
\hline Well & 1.000 & Reference & & 1.000 & Reference & \\
\hline Moderate/Poor & 3.114 & $1.793-5.408$ & $<0.001^{*}$ & 1.125 & $0.565-2.239$ & 0.738 \\
\hline \multicolumn{7}{|l|}{ VPI } \\
\hline Absent & 1.000 & Reference & & 1.000 & Reference & \\
\hline Present & 2.100 & $1.099-4.011$ & $0.025^{\star}$ & 1.184 & $0.495-2.835$ & 0.704 \\
\hline \multicolumn{7}{|l|}{ Location } \\
\hline Right & 1.000 & Reference & & & & \\
\hline Left & 0.874 & $0.488-1.565$ & 0.651 & & & \\
\hline \multicolumn{7}{|l|}{ Tumor diameter } \\
\hline \multicolumn{7}{|l|}{ TNM stage } \\
\hline $\mathrm{IA}$ & 1.000 & Reference & & 1.000 & Reference & \\
\hline IB & 2.100 & $1.212-3.636$ & $0.008^{\star}$ & 1.434 & $0.712-2.889$ & 0.313 \\
\hline \multicolumn{7}{|c|}{ Preoperative CEA level } \\
\hline$<10.0 \mathrm{ng} / \mathrm{mL}$ & 1.000 & Reference & & 1.000 & Reference & \\
\hline$\geq 10.0 \mathrm{ng} / \mathrm{mL}$ & 2.676 & $1.464-4.891$ & $<0.001^{*}$ & 1.930 & $0.985-3.780$ & 0.055 \\
\hline \multicolumn{7}{|c|}{ Postoperative CEA groups } \\
\hline NN group & 1.000 & Reference & & 1.000 & Reference & \\
\hline HN group & 3.241 & $0.745-14.101$ & 0.117 & 2.340 & $0.518-10.581$ & 0.269 \\
\hline $\mathrm{NH}$ group & 6.679 & $3.521-12.668$ & $<0.001^{*}$ & 4.672 & $2.294-9.514$ & $<0.001^{*}$ \\
\hline $\mathrm{HH}$ group & 8.644 & $3.915-19.083$ & $<0.001^{*}$ & 10.266 & $4.452-23.673$ & $<0.001^{*}$ \\
\hline
\end{tabular}

Longitudinal change of postoperative CEA: NN, continuously normal; $\mathrm{HN}$, increase first and then decrease; $\mathrm{NH}$, decrease first and then increase; $\mathrm{HH}$, continuously high. * $\mathrm{P}<0.05$. LUAD, lung adenocarcinoma; CEA, carcinoembryonic antigen; VPI, visceral pleural invasion; RFS, recurrence-free survival; HR, hazard ratio; Cl, confidence interval; TNM, tumor-node-metastasis; LUAD, lung adenocarcinoma. 

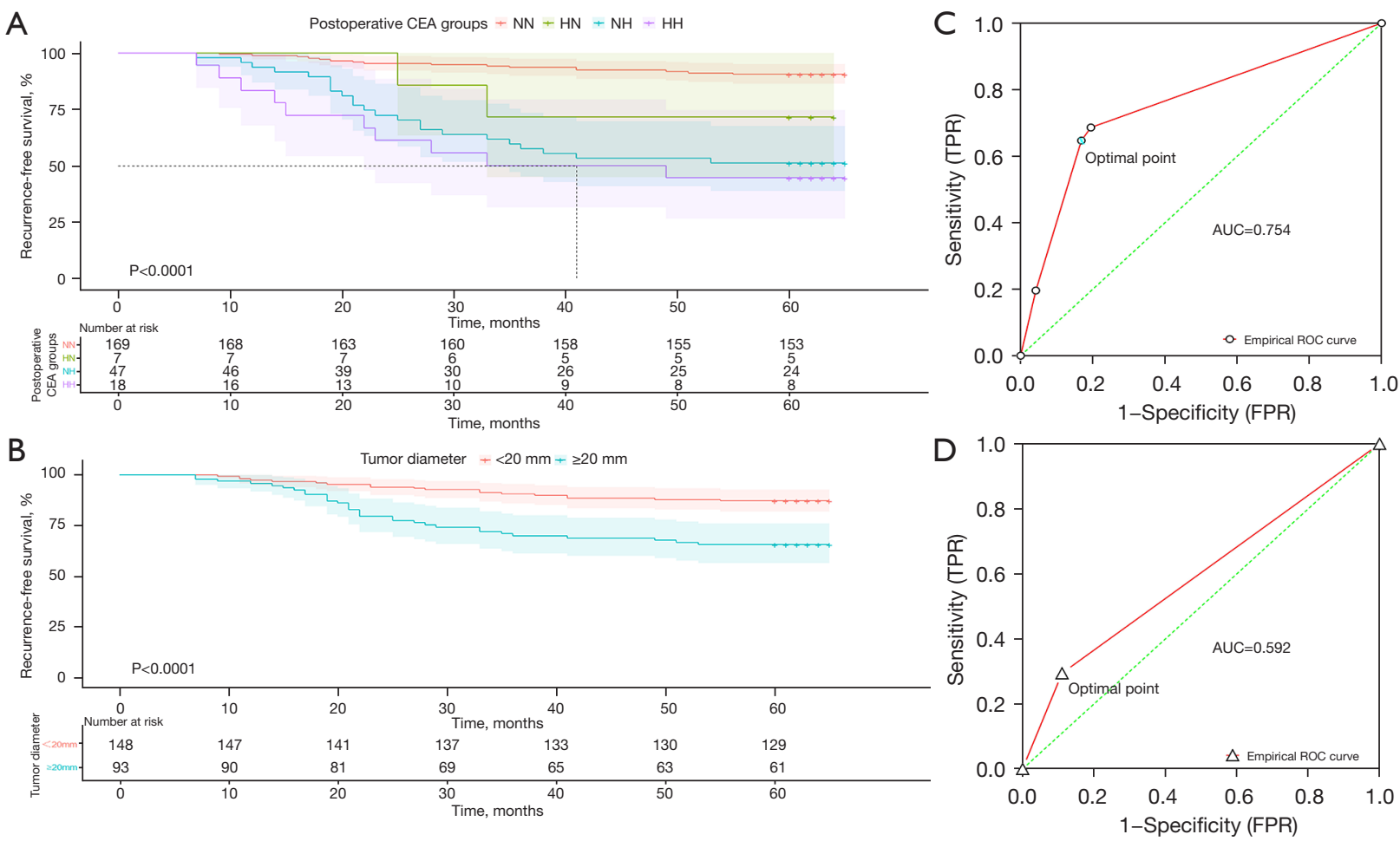

Figure 2 Postoperative CEA group and tumor diameter as independent predictors of RFS. (A) Kaplan-Meier curves showing RFS in 241 stage I LUAD patients stratified by postoperative CEA groups (NN, HN, NH and HH). (B) Kaplan-Meier curves showing RFS in 241 stage I LUAD patients stratified by tumor diameter ( $\geq 20$ and $<20 \mathrm{~mm}$ ). (C) ROC curve analysis was used to predict disease recurrence in 241 stage I LUAD patients according to postoperative CEA groups, and the AUC was 0.754. (D) ROC curve analysis was used to predict disease recurrence in 241 stage I LUAD patients according to preoperative serum CEA level, and the AUC was 0.592. Longitudinal change of postoperative CEA: NN, continuously normal; HN, increase first and then decrease; NH, decrease first and then increase; $\mathrm{HH}$, continuously high. ROC, receiver operating characteristic; AUC, area under the ROC curve; CEA, carcinoembryonic antigen; RFS, recurrence-free survival; LUAD, lung adenocarcinoma; FPR, false positive rate.

pancreatic cancer (25), renal pelvis adenocarcinoma (26), and breast cancer (27), and its high expression (positivity) has been significantly associated with worse prognosis. However, for the prediction of NSCLC, preoperative CEA level is controversial. Hanagiri et al. (41) indicated that for NSCLC patients, preoperative elevation of CEA is not associated with unfavorable survival. In our study, we found through univariate analysis that high preoperative CEA level was significantly associated with unfavorable survival in patients with stage I LUAD completely resectable by VATS (HR: 2.676; 95\% CI: 1.464-4.891; $\mathrm{P}<0.001$ ), but the sensitivity was only $29.4 \%$, which was consistent with previous research $(5,42)$. Lower sensitivity might lead to a higher omission rate (false-negative rate) and thus could not be found in stage I LUAD patients with recurrence potential. This may perhaps lead to a different prognosis for patients with completely resected stage I LUAD.

To our knowledge, this is the first study to predict the postoperative RFS of patients with surgically resected stage I LUAD using longitudinal changes in serum CEA level before and after surgery. We divided the levels of the longitudinal changes of serum CEA tested twice after the operation into different postoperative CEA groups (NN group, HN group, $\mathrm{NH}$ group, and $\mathrm{HH}$ group). We found that patients in the NN group and the HN group had better RFS, while those in the $\mathrm{NH}$ and $\mathrm{HH}$ groups had poor outcomes.

In our study, it was shown that smaller tumor diameter (<20 mm: HR: $2.437,95 \%$ CI: $1.245-4.770, \mathrm{P}=0.009)$ had a significant effect on stage I LUAD survival (AUC $=0.592$ ), which is consistent with a few previous studies (5,43-45). Moreover, we found that postoperative CEA grouping (NN vs. $\mathrm{NH}, \mathrm{NN}$ vs. $\mathrm{HH}$; both $\mathrm{P}<0.001$ ) had a considerable 
A

Points
Diameter
Postoperative CEA groups
Total Points
1-year recurrence-free survival
3-year recurrence-free survival
5-year recurrence-free survival

B

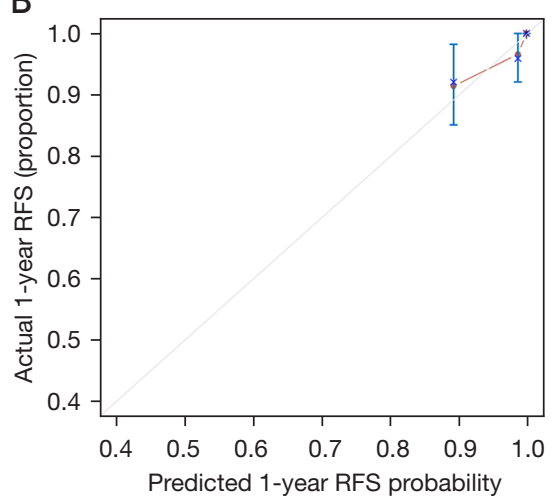

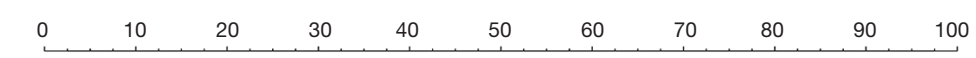

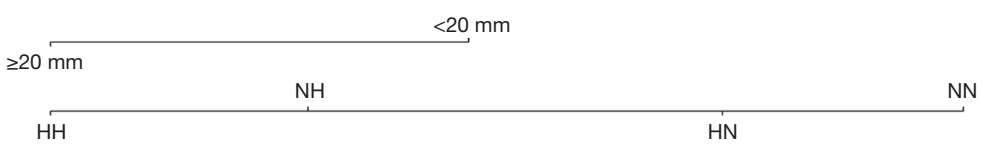

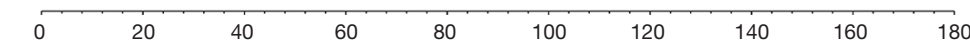

3

$\begin{array}{llllllll}3.5 & 4 & 4.5 & 5 & 5.5 & 6 & 6.5\end{array}$

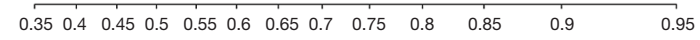

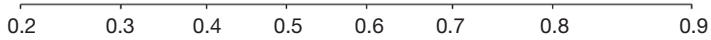

C

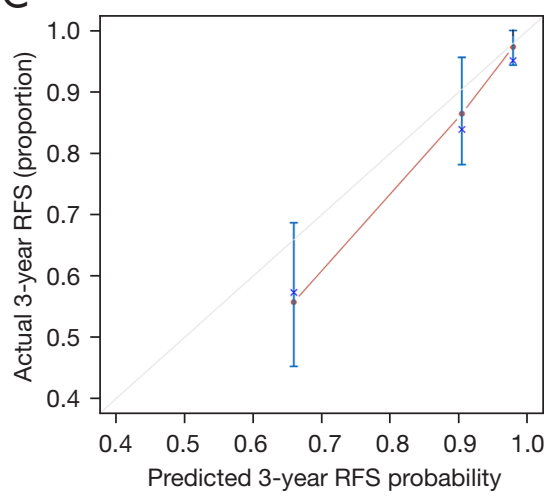

$\mathrm{D}$

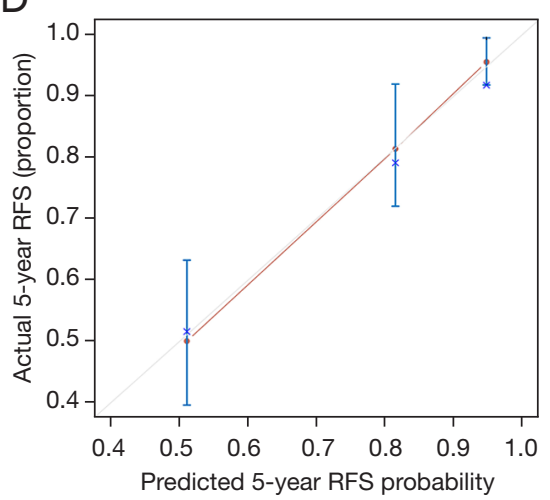

Figure 3 Nomogram and calibration curves. (A) A nomogram to predict recurrence of stage I LUAD was drawn based on the multivariate Cox regression model. (B) Calibration curve of the nomogram for 1-year RFS. (C) Calibration curve of the nomogram for 3-year RFS. (D) Calibration curve of the nomogram for 5-year RFS. Longitudinal change of postoperative CEA: NN, continuously normal; HN, increase first and then decrease; NH, decrease first and then increase; HH, continuously high. CEA, carcinoembryonic antigen; RFS, recurrence-free survival; LUAD, lung adenocarcinoma.

effect on the recurrence of stage I LUAD patients. Many of the recent studies on this subject have concentrated on either the predictive effect of the preoperative serum CEA levels on the prognosis of patients or the combination of serum CEA with other markers for diagnosis (31-36). In these latter combination studies, a universal cutoff value or the reference value of the kit was used. However, a universal cutoff value cannot be fully applied to each individual patient, as this does not account for individual differences. Other studies have also considered the relationship between the preoperative serum CEA level and the serum CEA level measured the first time after surgery $(5,36,41)$; however, this can only determine whether the patient improved after surgical removal of the tumor lesions compared with before the operation. The occurrence and development of lung cancer is a process of longitudinal change, and this dynamic must also be considered. Previous large-scale clinical studies have found that high CEA levels in patients before surgery generally decrease after surgery, regardless of the surgery's success or the presence of early postoperative recurrence (44). Even after successful complete resection of the tumor at an early stage, the patient's 5-year RFS was still found to be relatively high $(15,46)$, suggesting the presence of prognostic factors other than disease stage and the micrometastases of developing tumor cells. Thus, distinguishing between a high risk and low risk of recurrence after surgery may be difficult. Consequently, we have proposed the concept of longitudinal changes in serum CEA level after surgery and divided the level of the longitudinal changes of serum CEA tested twice after the operation into different postoperative 
CEA groups: NN group (continuously normal), HN group (increase first and then decrease), NH group (decrease first and then increase,), and $\mathrm{HH}$ group (continuously high). We also referred to the manufacturer's guidance regarding the level of preoperative serum CEA, but we focused on the longitudinal changes of serum CEA.

Through Cox regression analysis, our data suggested that patients in the $\mathrm{NH}$ group $(\mathrm{P}<0.001)$ and $\mathrm{HH}$ group $(\mathrm{P} \leq 0.001)$ had poor prognosis, and their 5 -year RFS rates were $50.1 \%$ and $44.4 \%$, respectively. We suspected that the failure to reach the normal CEA level or the increase in CEA may be caused by occult MRD, which is already present throughout the body during surgery and cannot be detected. This indicates that the true tumor stage was underestimated. For the HN group (increase first and then decrease), our speculation was that, although the surgery could completely remove the tumor foci, the tumor cells entered the systemic circulation before this, leading to an increase in serum CEA. Eventually they were eliminated by the immune system because they could not form new foci, resulting in a decrease in serum CEA. Moreover, the treatment of the tumor itself during surgery may lead to the spread of cancer cells (47). We also found that postoperative CEA grouping has a good predictive value for predicting postoperative recurrence and metastasis of stage I LUAD (AUC $=0.754$ ). Based on the RFS of the stage I LUAD patients, we established a risk scoring system to predict the recurrence risk of stage I LUAD after surgery. The C-index value was 0.789 and was verified by calibration, indicating that this could indeed predict the patient's RFS.

Longitudinal change also has predictive value for stage III-IV LUAD. However, patients with stage III-IV LUAD generally do not undergo surgery. So, we did not discuss it in our study. Furthermore, Common resection has greater surgical damage for patients, than single-port VATS, which might affect the serum CEA level. We would need to further explore and research.

There are some limitations in this study. First, this was a single-center retrospective study, and selection bias was unavoidable. Second, there was a small sample size in the HN group ( $n=7)$ and observed events, making statistical interpretation of the results difficult. Additional multicenter studies with larger patient cohorts may address these limitations.

\section{Conclusions}

Our results showed longitudinal changes in serum
CEA levels after single-port VATS was an independent unfavorable prognostic factor in completely resected stage I LUAD patients. Postoperative CEA groups (NH /HH group $v s$. NN/HN group) were significantly associated with worse RFS. This study established a nomogram to predict the postoperative recurrence of patients with stage I LUAD, which can be used for the early monitoring of postoperative recurrence in these patients.

\section{Acknowledgments}

The authors appreciate the academic support from AME Lung Cancer Collaborative Group.

Funding: This study was supported by Shanghai Pulmonary Hospital Research Fund (No. FKLY20003).

\section{Footnote}

Reporting Checklist: The authors have completed the TRIPOD reporting checklist. Available at https://dx.doi. org/10.21037/tlcr-21-833

Data Sharing Statement: Available at https://dx.doi. org/10.21037/tlcr-21-833

Conflicts of Interest: All authors have completed the ICMJE uniform disclosure form (available at https://dx.doi. org/10.21037/tlcr-21-833). The authors have no conflicts of interest to declare.

Ethical Statement: The authors are accountable for all aspects of the work in ensuring that questions related to the accuracy or integrity of any part of the work are appropriately investigated and resolved. The study was conducted in accordance with the Declaration of Helsinki (as revised in 2013). The study was approved by Shanghai Pulmonary Hospital Institutional Review Board (No. KY2020-1), and individual consent for this retrospective analysis was waived.

Open Access Statement: This is an Open Access article distributed in accordance with the Creative Commons Attribution-NonCommercial-NoDerivs 4.0 International License (CC BY-NC-ND 4.0), which permits the noncommercial replication and distribution of the article with the strict proviso that no changes or edits are made and the original work is properly cited (including links to both the formal publication through the relevant DOI and the license). 
See: https://creativecommons.org/licenses/by-nc-nd/4.0/.

\section{References}

1. Siegel RL, Miller KD, Jemal A. Cancer statistics, 2020. CA Cancer J Clin 2020;70:7-30.

2. Wu C, Li M, Meng H, et al. Analysis of status and countermeasures of cancer incidence and mortality in China. Sci China Life Sci 2019;62:640-7.

3. National Comprehensive Cancer Network. Non-Small Cell Lung Cancer (Version 4. 2021). Available online: https://www.nccn.org/professionals/physician_gls/default. aspx (accessed on 6 May 2021).

4. Zhang Y, Ma X, Shen X, et al. Surgery for pre- and minimally invasive lung adenocarcinoma. J Thorac Cardiovasc Surg 2020. [Epub ahead of print].

5. He Y, Cui Y, Chang D, et al. Postoperative CYFRA 21-1 and CEA as prognostic factors in patients with stage I pulmonary adenocarcinoma. Oncotarget 2017;8:73115-22.

6. Uramoto H, Tanaka F. Prediction of recurrence after complete resection in patients with NSCLC. Anticancer Res 2012;32:3953-60.

7. Isaka M, Kojima H, Takahashi S, et al. Risk factors for local recurrence after lobectomy and lymph node dissection in patients with non-small cell lung cancer: Implications for adjuvant therapy. Lung Cancer 2018;115:28-33.

8. Yuan Q, Wang W, Zhang Q, et al. Clinical Features and Prognostic Factor of Thoracic Postoperative OligoRecurrence of Non-Small-Cell Lung Cancer. Cancer Manag Res 2020;12:1397-403.

9. Sekihara K, Hishida T, Yoshida J, et al. Long-term survival outcome after postoperative recurrence of non-small-cell lung cancer: who is 'cured' from postoperative recurrence? Eur J Cardiothorac Surg 2017;52:522-8.

10. al-Kattan K, Sepsas E, Fountain SW, et al. Disease recurrence after resection for stage I lung cancer. Eur J Cardiothorac Surg 1997;12:380-4.

11. Sun K, You A, Wang B, et al. Clinical T1aN0M0 lung cancer: differences in clinicopathological patterns and oncological outcomes based on the findings on high-resolution computed tomography. Eur Radiol 2021;31:7353-62.

12. Wu HL, Wu YM, Chen JT, et al. A comparison of inflammation markers for predicting oncological outcomes after surgical resection of non-small-cell lung cancer: a validated analysis of 2,066 patients. Sci Rep 2020;10:19523.

13. Guibert N, Pradines A, Favre G, et al. Current and future applications of liquid biopsy in nonsmall cell lung cancer from early to advanced stages. Eur Respir Rev 2020;29:190052.

14. MRD May Predict Relapse in NSCLC. Cancer Discov 2020;10:OF7.

15. Okami J, Shintani Y, Okumura M, et al. Demographics, Safety and Quality, and Prognostic Information in Both the Seventh and Eighth Editions of the TNM Classification in 18,973 Surgical Cases of the Japanese Joint Committee of Lung Cancer Registry Database in 2010. J Thorac Oncol 2019;14:212-22.

16. Wood DE, Kazerooni EA, Baum SL, et al. Lung Cancer Screening, Version 3.2018, NCCN Clinical Practice Guidelines in Oncology. J Natl Compr Canc Netw 2018;16:412-41.

17. Rampinelli C, De Marco P, Origgi D, et al. Exposure to low dose computed tomography for lung cancer screening and risk of cancer: secondary analysis of trial data and riskbenefit analysis. BMJ 2017;356:j347.

18. Sadate A, Occean BV, Beregi JP, et al. Systematic review and meta-analysis on the impact of lung cancer screening by low-dose computed tomography. Eur J Cancer 2020;134:107-14.

19. Backhus LM, Farjah F, Zeliadt SB, et al. Predictors of imaging surveillance for surgically treated early-stage lung cancer. Ann Thorac Surg 2014;98:1944-51; discussion 1951-2.

20. Srikantharajah D, Ghuman A, Nagendran M, et al. Is computed tomography follow-up of patients after lobectomy for non-small cell lung cancer of benefit in terms of survival? Interact Cardiovasc Thorac Surg 2012;15:893-8.

21. Brenner DJ, Hall EJ. Computed tomography--an increasing source of radiation exposure. $\mathrm{N}$ Engl J Med 2007;357:2277-84.

22. Swensen SJ, Jett JR, Hartman TE, et al. CT screening for lung cancer: five-year prospective experience. Radiology 2005;235:259-65.

23. Berinstein NL. Carcinoembryonic antigen as a target for therapeutic anticancer vaccines: a review. J Clin Oncol 2002;20:2197-207.

24. Deng K, Yang L, Hu B, et al. The prognostic significance of pretreatment serum CEA levels in gastric cancer: a meta-analysis including 14651 patients. PLoS One 2015;10:e124151.

25. Wu L, Huang P, Wang F, et al. Relationship between serum CA19-9 and CEA levels and prognosis of pancreatic cancer. Ann Transl Med 2015;3:328.

26. Ye YL, Bian J, Huang YP, et al. Primary mucinous 
adenocarcinoma of the renal pelvis with elevated CEA and CA19-9. Urol Int 2011;87:484-8.

27. Wang $W, \mathrm{Xu} X$, Tian B, et al. The diagnostic value of serum tumor markers CEA, CA19-9, CA125, CA153 , and TPS in metastatic breast cancer. Clin Chim Acta 2017;470:51-5.

28. Matsuoka K, Sumitomo S, Nakashima N, et al. Prognostic value of carcinoembryonic antigen and CYFRA21-1 in patients with pathological stage I non-small cell lung cancer. Eur J Cardiothorac Surg 2007;32:435-9.

29. Vincent RG, Chu TM, Fergen TB, et al. Carcinoembryonic antigen in 228 patients with carcinoma of the lung. Cancer 1975;36:2069-76.

30. Vincent RG, Chu TM, Lane WW. The value of carcinoembryonic antigen in patients with carcinoma of the lung. Cancer 1979;44:685-91.

31. Moritz R, Muller M, Korse CM, et al. Diagnostic validation and interpretation of longitudinal circulating biomarkers using a biomarker response characteristic plot. Clin Chim Acta 2018;487:6-14.

32. Zheng J, Ye X, Liu Y, et al. The combination of CTCs and CEA can help guide the management of patients with SPNs suspected of being lung cancer. BMC Cancer 2020;20:106.

33. Zhou J, Diao X, Wang S, et al. Diagnosis Value of Combined Detection of Serum SF, CEA and CRP in Non-Small Cell Lung Cancer. Cancer Manag Res 2020;12:8813-9.

34. Yang Q, Zhang $\mathrm{P}, \mathrm{Wu} \mathrm{R}$, et al. Identifying the Best Marker Combination in CEA, CA125, CY211, NSE, and SCC for Lung Cancer Screening by Combining ROC Curve and Logistic Regression Analyses: Is It Feasible? Dis Markers 2018;2018:2082840.

35. de Jong C, Deneer VHM, Kelder JC, et al. Association between serum biomarkers CEA and $\mathrm{LDH}$ and response in advanced non-small cell lung cancer patients treated with platinum-based chemotherapy. Thorac Cancer 2020;11:1790-800.

36. Sone K, Oguri T, Ito K, et al. Predictive Role of CYFRA21-1 and CEA for Subsequent Docetaxel in Non-small Cell Lung Cancer Patients. Anticancer Res 2017;37:5125-31.

37. Konishi T, Shimada Y, Hsu M, et al. Association of Preoperative and Postoperative Serum Carcinoembryonic Antigen and Colon Cancer Outcome. JAMA Oncol 2018;4:309-15.

38. Jing R, Cui M, Ju S, et al. The Changes and Clinical
Significance of Preoperative and Postoperative Serum CEA and CA19-9 in Gastric Cancer. Clin Lab 2020.

39. Mu W, Jiang L, Zhang J, et al. Non-invasive decision support for NSCLC treatment using PET/CT radiomics. Nat Commun 2020;11:5228.

40. Gold P, Freedman SO. Specific carcinoembryonic antigens of the human digestive system. J Exp Med 1965;122:467-81.

41. Hanagiri T, Sugaya M, Takenaka M, et al. Preoperative CYFRA 21-1 and CEA as prognostic factors in patients with stage I non-small cell lung cancer. Lung Cancer 2011;74:112-7.

42. Ma S, Shen L, Qian N, et al. The prognostic values of CA125, CA19.9, NSE, AND SCC for stage I NSCLC are limited. Cancer Biomark 2011;10:155-62.

43. Cao X, Zheng YZ, Liao HY, et al. A clinical nomogram and heat map for assessing survival in patients with stage I non-small cell lung cancer after complete resection. Ther Adv Med Oncol 2020;12:1758835920970063.

44. Okada M, Nishio W, Sakamoto T, et al. Prognostic significance of perioperative serum carcinoembryonic antigen in non-small cell lung cancer: analysis of 1,000 consecutive resections for clinical stage I disease. Ann Thorac Surg 2004;78:216-21.

45. Kozu Y, Maniwa T, Takahashi S, et al. Prognostic significance of postoperative serum carcinoembryonic antigen levels in patients with completely resected pathological-stage I non-small cell lung cancer. J Cardiothorac Surg 2013;8:106.

46. Pasini F, Pelosi G, Valduga F, et al. Late events and clinical prognostic factors in stage I non small cell lung cancer. Lung Cancer 2002;37:171-7.

47. Hashimoto M, Tanaka F, Yoneda K, et al. Significant increase in circulating tumour cells in pulmonary venous blood during surgical manipulation in patients with primary lung cancer. Interact Cardiovasc Thorac Surg 2014;18:775-83.

Cite this article as: Chen $\mathrm{H}$, Jiang Y, Jia K, Zhang K, Matsuura N, Jeong JY, Su B, Zhou X. Prognostic significance of postoperative longitudinal change of serum carcinoembryonic antigen level in patients with stage I lung adenocarcinoma completely resected by single-port video-assisted thoracic surgery: a retrospective study. Transl Lung Cancer Res 2021;10(10):3983-3994. doi: 10.21037/tlcr-21-833 